\title{
Developing Physical Axiomatics: Results and Outcomes
}

\author{
Maria Astafurova ${ }^{1, *}$ \\ ${ }^{1}$ HSE University, Faculty of Economic Sciences, RU-101000, Moscow, Russia
}

\begin{abstract}
The ideology and scheme of physical axiomatics are proposed, the initial postulates and axioms are formulated. The essential principles of axiomatics are based on scientific statements and concepts that reflect the fundamental properties of the physical world. One of the obtained outcomes is the statement: "In the physical world, all homogeneous quantities are commensurable, and every real physical property is limited by a certain ; characteristic for this property, minimum nonzero value". Homogeneous quantities are quantities of one and the same nature, relating to the manifestation of one and the same physical property. The analysis of the problem of incommensurability of radius and circumference leads to the conclusion about the existence of a fundamental property of the physical world, different from space and time. This property is manifested in the forms of electric charges, electric and magnetic fields, electromagnetic waves and fields. Given the nature of its manifestation, this property is called the "electromagnetic property". The developed axiomatic system and the obtained consequences are the basis of the working model of the physical vacuum. On the basis of this model there have been derived the equations connecting parameters of basic interactions. The components of the internal energy of the physical vacuum are determined. One of these components determines the phenomenon of the Universe expansion.
\end{abstract}

\section{Introduction}

The task of scientists engaged in the basics of any science - to bring the original scientific provisions (definitions, postulates, axioms) to the reality of the world. The more correct and complete original axiomatics will display the properties of the physical world, the more correct and complete will be the scientific results and conclusions.

Studies by the English philosopher Bertrand Russell (1872-1970) and the Austrian mathematician Kurt Gödel (1906-1978) showed that the creating an ideal axiomatic construction not containing internal contradictions is theoretically impossible.

This conclusion restricts the possibility of creating axiomatic foundations of mathematics, physics and separate sections of these sciences, but does not reduce the importance of such constructions.

Earlier, we proposed to expand the axiomatics of mathematics, supplementing it with scientific support illustrating the fundamental properties of the physical world [1]. It was assumed that this extension of axiomatics will increase the reliability of mathematical models and will allow obtaining new scientific results. Developing extended axiomatics of mathematics was considered to be an important step towards solving Hilbert's sixth problem "Mathematical exposition of the foundations of physics".

David Hilbert (1862-1943) identified two major issues of the problem of constructing the mathematical axiomatics of physics:

- axiomatization of probability theory as the Foundation of statistical physics;
- axiomatization of the theory which will connect atomistic representations with laws of motion of continuums.

The axiomatics of probability theory was constructed by A.N. Kolmogorov (1903-1987) in 1929-1933 [2].

The solution of the second part of the problem posed by Hilbert is still problematic. The analysis of this problem is presented in [3-6].

Many researchers considered and analyzed the possibility of constructing axiomatic foundations of physics and its separate sections, for example [7, 8]. The desire to deduce the observed physical phenomena from "clear and distinct ideas" (primary principles, primary causes) can be found in the works of René Descartes.

From our point of view, mathematics and physics should have an equal ideology of axiomatic model based on the concepts acquired by man in the process of practical experience. And we find confirmation of this thesis in the words of the Russian mathematician V.I. Arnold (1937-2010): "Mathematics is an experimental part of theoretical physics and a member of the family of natural sciences" [9].

\section{Problem statement}

If we set the task to develop physical axiomatics, we must first of all determine the object of our action.

The science of physics is a heterogeneous scientific field of knowledge. It consists of many sections covering different types of movements and different processes of micro- and macro-worlds. Physics is a huge scientific tree, the roots of which nourish accurate knowledge of all branches of natural sciences. However, the initial and

e-mail: astafurova.maria@mail.ru 
defining task of physics is the formation of the ideological foundation of natural science as a whole.

We consider the problem of developing the axiomatic foundations of physics as creationg a complex of axiomatic foundations of all its sections.

The first step towards solving this problem, in our opinion, should be developing axiomatic foundations of the initial section of physics, which considers and defines the most general ideas and concepts about the world, forming the worldview basis of natural science.

Having determined the object of our action, we proceed to build the desired axiomatic system.

\section{Definitions}

We use the following definitions:

- The physical world is everything that exists.

- A physical object is an object of the physical world.

- Motion is a change in the relative position of physical objects or parts of a physical object.

- Postulate - statement (position), accepted as truth, without proof, reflecting some fundamental (fundamental) property of the physical world.

- An axiom is a statement accepted as an obvious fact without proof, reflecting some particular property of the physical world.

- A theorem is a statement that requires proof and is derived from original postulates, axioms, and already proven theorems.

- A consequence is a statement that follows logically from a postulate, axiom, proven theorem, or experimental fact.

\section{The original system of postulates and axioms}

Let us introduce into physical axiomatics the following scientific propositions reflecting the fundamental properties of the physical world into the physical axiomatics:

- Postulate P1. The physical world does not contain emptiness. Any part of the physical world is filled with some physical entity;

- Postulate P2. Movement is an inherent property of the physical world;

- Postulate P3. Space, or extension - is an inherent property of the physical world;

- Axiom A1. Wave motion is a universal form of motion.

- Axiom A2. Every property of the physical world in quantitative terms can be represented by a number of finite value, that is, a rational number.

Axioms A1 and A2 are not self-evident. Therefore, we present the rationale for their introduction into physical axiomatics.

\subsection{Justification of the introduction of axiom A1}

Consideration and analysis of various forms and types of motion shows that any motion is a wave process. This process affects all sides and faces of the moving object.
Its external manifestation is an oscillating change in all values and parameters characterizing the object. In any system of the physical world, regardless of its nature, size and hierarchical status, there are continuous fluctuations in the quantitative characteristics of all properties. Wave motion is a natural and universal form of motion. The physical world is a set of oscillating objects. This totality is perceived by us as an inseparable continuum of the physical world.

Axiom A1 is introduced into physical axiomatics based on the analysis of processes occurring in the microcosm, biosphere, macrocosm.

\subsection{Justification of the introduction of axiom A2}

The numerical value that we ascribe to any property of the physical world is always relative.

Measurement of any property consists of registration of indications of the measuring device. When measuring properties, the test and reference objects are compared. The value of the reference object property is always the number of the final values??? The reading of any instrument is a rational number. Therefore, the result of measuring any property is a rational number. We conclude that the magnitude of any property of the physical world can be expressed by a rational number.

\section{The consequences of the initial postulates and axioms}

\subsection{The consequence of the postulate P1}

The number "zero" does not represent emptiness at all, but the absence of anything in the object or phenomenon under consideration. The same is applied to an empty set.

Note. According to the postulate $\mathrm{P} 1$, the space of the physical world, which does not contain matter, is filled with some other physical entity. This physical entity in different scientific epochs was called: "emptiness", "empty space", "intangible substance", "world ether", "field", "quintessence", "cosmic vacuum" and "physical vacuum". Following the modern scientific tradition, we will call this physical entity a physical vacuum. It is quite obvious that matter and physical vacuum are interrelated forms of the physical world.

\subsection{The consequence of the postulate $P 2$}

The resting state does not exist and is a conditional model. Movement is inherent in any part of the physical world and manifests itself in the volume of any smallness.

Note. It is logical to assume that due to the universality and continuity of movement, in the physical world there should be a tendency to achieve a natural ratio of all parameters. That is, it can be expected that every natural movement directs the physical object to achieve some natural state. Is this state of the object optimal and harmonic? Is it possible to talk about the 
optimal state of a physical object in the case, for example, of a particle in the state of Brownian motion? Is "ordinary man" such a particle in a large socium? These are debatable issues that we bring to the table. These are controversial issues that we bring up for discussion.

\subsection{The outcome of the joint consideration of postulates $P 1$ and $P 3$}

The spatial size of a physical object cannot be zero.

Note. In modern physics, some objects of the microcosm, recognized as existing, are considered as having no spatial size. Therefore, such objects either do not exist in reality, or the idea of them requires revision. The zero value of the size of any really existing object of the physical world should be considered as a conditional model.

\subsection{The outcome of axiom A1}

Uniform and rectilinear motion does not exist in the physical world.

\subsection{The outcome of axiom A2}

All homogeneous quantities are commensurable and the ratio of homogeneous quantities is always a rational number.

Explanation. Homogeneous quantities are quantities of one and the same nature, relating to the manifestation of one physical property.

Note. The statement about commensurability of homogeneous quantities extends also to physical segments. However, the conclusion about the commensurability of any segments contradicts the results obtained on the basis of the Pythagorean theorem. According to the calculations made on the basis of this theorem, the length of the diagonal of a square can be expressed as an irrational number. This contradiction is imaginary. In the physical world, the scope of the Pythagorean theorem is limited. This is due to the curvature of the physical world and the existence of a minimum length.

\subsection{The outcome of the joint consideration of postulate $\mathrm{P} 1$ and axiom A2}

Each real physical property is limited by a certain characteristic for this property, minimum nonzero value. The zero value of the physical property should be considered to be a conditional model.

\section{Experimental and calculated data confirming the consequence $\mathbf{4 . 6}$}

Experimental confirmation of the consequence 4.6:

- Existence in the physical world of carriers of elementary (minimum) electric charge (electron and positron);
- Existence of "absolute zero" of the temperature (minus $273,15^{\circ} \mathrm{C}$ ). At this temperature, the vibrational motion of particles (the so-called "zero oscillations") is preserved in the physical body. That is, the concept of "absolute zero" of the temperature implies the existence of its minimum value other than zero.

\subsection{Determination of the minimum mass}

To calculate the numerical value of the minimum mass, we analyzed the mass spectrum of elementary particles [1].

This analysis allowed us to distinguish three groups of particles with fundamentally different rest mass: the photon and all kinds of neutrinos (zero rest mass); the electron (rest mass $0.511 \mathrm{MeV} / \mathrm{s}^{2}$ ); all other elementary particles (rest mass $\geq 105.66 \mathrm{MeV} / \mathrm{s}^{2}$ ). A comparison of these three groups led to the following conclusions:

- photon and neutrino should be considered not as elementary particles with zero rest mass, but as objects of the microcosm, which have no rest mass property; - the physical nature of the mass of the electron is different from the physical nature of the mass of all other elementary particles;

- all elementary particles (without an electron), regardless of the difference in their properties, can be considered as a set of objects with the same physical nature of their rest mass.

The analysis of the selected set of homogeneous quantities characterizing the rest mass of elementary particles allowed us to determine the numerical value of the minimum mass equal to $34.75 \mathrm{MeV} / \mathrm{s}^{2}$.

\subsection{Determination of the minimum length}

The issue of the existence, in the physical world, of a universal constant with the dimension of length has been repeatedly discussed in the literature. Values from $10^{-19}$ $\mathrm{m}$ to $10^{-35} \mathrm{~m}$ are proposed for the minimum length.

To calculate the numerical value of the minimum length, we analyzed the experimental data characterizing the sizes of micro - and macro-objects of the physical world [1]. The numerical value of the minimum length $\left(\sim 10^{-17} \mathrm{~m}\right)$ was determined by the graphical method using the extrapolation method.

\subsection{About minimum time}

Consequence 4.6 asserts the existence in the physical world of minimum values other than zero of all real physical properties.

For the property "electric charge" this statement is quite obvious from the point of view of experimental data. For some other properties (temperature, energy, mass, length) - this statement can be theoretically justified. As for time, the formulation of the problem of determining its minimum value is, in fact, a new problem of theoretical physics.

In relation to the property of time, corollary 4.6 means that there is no "instantaneity" of events. Also, this consequence imposes certain restrictions on 
Newton's third law, since action and counteraction cannot be simultaneous. Opposition to the acting force is separated from the process of action by a certain time interval, a multiple of the minimum time.

\section{Analysis of the problem of incommensurability of radius and circumference and the conclusion about the existence of a fundamental property of the physical world, different from space and time}

According to consequence 4.5 all homogeneous quantities are commensurable, and the ratio of homogeneous quantities is always a rational number.

However, the length of the radius and the length of the circle are incommensurable. How to explain this fact?

In mathematics, length is considered to be a certain extent, regardless of the physical nature of the object under consideration (measured). The length of a segment, an arc, a circle, any other curvilinear line is understood in mathematics uniformly - as a well-defined extension, as "length in general".

Is this mathematical abstraction fully applicable to the objects of the physical world? In other words, are the values of the length of the segment and the length of the arc homogeneous quantities that have the same physical nature? The incommensurability of the length of the radius and the length of the circle shows that these values must be considered as a manifestation of different properties of the physical world.

Let us assume a priori that the radius (and, in general, the size of any physical object) is due to the manifestation of the property of space. Then the circle, as well as the sphere of which the circle is a crosssection, all other curvilinear lines and figures of rotation, as well as waves, is due to the manifestation of some other property of the physical world. It is this property that determines the universality of wave motion and the formation of bodies of rotation of the physical world.

Thus, the analysis of the problem of incommensurability of radius and circle leads to the conclusion about the existence of a fundamental property of the physical world, different from space and responsible for the formation of bodies of rotation [10]. It is obvious that this property is not time. Time and the space-time continuum of Hermann Minkowski cannot be the source and cause of wave motion and the formation of spherical bodies of the physical world.

\section{Electromagnetic property of the physical world and postulate P4}

Let's pay attention to such physical phenomena as electromagnetic waves, electric charge, magnetic and electric field.

These phenomena, in one form or another, manifest themselves in every object and process of the physical world. It is obvious that all these phenomena are caused by the manifestation of some fundamental property of the physical world. Given the nature of its manifestation, we will call this property "electromagnetic property".

We introduce a new postulate into physical axiomatics:

- Postulate P4: Electromagnetic property is an inherent property of the physical world.

\subsection{The consequence of postulates P3, P4 and axiom A1}

The physical world is a wave spatial-electromagnetic continuum.

\section{Spatial-electromagnetic model of physical vacuum and its applications}

Consequence 7.1 is the basis of the spatialelectromagnetic model of the physical vacuum (and the physical world as a whole) [11].

Space (extension) and electromagnetic property are considered in the model as attributes and integral properties of the physical world. Matter and physical vacuum are interrelated forms of the physical world.

The physical vacuum is represented in the model as a wave vector continuum. A spatial vector characterizes space. The electromagnetic vector characterizes the electromagnetic property of the physical world. The number of components of the spatial vector corresponds to the dimensionality of the observed space. The number of components of the electromagnetic vector corresponds to two types of electric charges and magnetic poles.

Taking into account the thesis about the universality of wave motion, the physical world is also considered as a set of interconnected oscillators. The smallest natural oscillator is the unit cell of the physical vacuum.

The new model opens up the possibility of solving a number of fundamental problems in the field of natural science, which previously could not be solved.

In particular, on the basis of the spatialelectromagnetic model, the following problems are solved:

- The equations connecting spatial parameters of fundamental hierarchical structures and force parameters of fundamental interactions with properties of physical vacuum are derived [11]. The calculated values of the parameters are in agreement with the experimental data;

- Components of internal energy of physical vacuum are defined, analytical expressions for each of these components are established and their numerical values are calculated. One of these components, caused by the properties of space, is associated with the phenomenon of expansion of the space of the Universe [12]. It is also shown that the nature of the inert mass is interrelated with the properties of space and is caused by it.

The new working model of physical vacuum has predictive power. From the spatial-electromagnetic model, it is necessary to conclude that there is spatial radiation in the physical world. Oscillations of the electromagnetic component of the structure of the physical vacuum lead to the formation of 
electromagnetic waves, and oscillations of its spatial component lead to the formation of spatial waves. It is proposed to consider neutrinos as a quantum of spatial radiation.

\section{References}

1. M.V. Astafurova, Experience of construction of the physical axioms of mathematics (FON-SCIENCE, Bugul'ma, Russia, 2013)

2. A.N. Kolmogorov, Grundbegriffe der Wahrscheinlichkeitsrechnung (SpringerVerlag, Berlin, 1933)

3. L. Corry, Arch. Hist. Exact Sci. 51, 83 (1997)

4. L. Saint-Raymond, Hydrodynamic limits of the Boltzmann equation (Springer-Verlag, Berlin, 2009)

5. M. Slemrod, Comput. Math. Appl. 65, 1497 (2013)

6. A.N. Gorban, I. Karlin, Bull. Amer. Math. Soc. 51, 186 (2014)

7. H. Reichenbach, Axiomatik der relativistischen Raum-Zeit-Lehre (Fried. Vieweg \& Sohn, Braunschweig, 1924)

8. M. Bunge, Philosophy of Physics (D. Reidel Publ. Comp., Dordrecht, 1973)

9. V.I. Arnold, "Hard" and "Soft" mathematical models (MTsNMO, Moscow, 2000)

10. M.V. Astafurova, V.I. Astafurov, Educational resources and technologies, 4, 256 (2014)

11. M.V. Astafurova, S.L. Dobretsov, V.I. Astafurov, FON-SCIENCE, 38, 5 (2014)

12. M.V. Astafurova, Proc. 14th Int. Conf. "Zababakhin scientific talks” (VNIITF, Snezhinsk, Russia, 2019) 